including insulin, glucagon, and somatostatin. However, the authors also show that the hypertension seen in the high-salt-fed PC2-knockout animals can be corrected by administration of a synthetic $\gamma$-MSH analogue and that the same results are seen in mice in which the $\gamma$-MSH receptor, melanocortin receptor 3 (MC3-R), is knocked out. So why hasn't $\gamma$-MSH been implicated in salt-sensitive hypertension until now? Part of the reason is certainly the multitude of neuroendocrine signals mediated by the other peptides produced along with $\gamma$-MSH when POMC is processed (Figure 1), making it hard to isolate $\gamma$-MSH-specific actions in a physiological setting. In fact, 5 receptors have been identified to date - MC1-R, MC2-R, MC3-R, MC4-R, and MC5-R - for the melanocortin hormones alone, with more possibly to come. These receptors have a broad tissue distribution and they mediate a range of physiological responses depending on their location. These include: (a) pigmentation (MC1-R); (b) modulation of corticosterone levels (MC2-R); (c) appetite suppression and metabolic activation (MC4-R); (d) thermoregulation and water repulsion (MC5-R); and (e) inflammation (MC1-R and MC3-R). It's easy to imagine that several of these responses might have masked or complicated the analysis of blood pressure effects of $\gamma-\mathrm{MSH}$.

Does this mean that $\gamma-\mathrm{MSH}$ is the cause of salt-induced hypertension in humans? Not by a long shot! The knockout of other mouse genes, including those for atrial natriuretic peptide (6) and its receptor (7), the prostaglandin receptor EP2 (8), and the bradykinin receptor (9), also leads to salt-sensitive hypertension. However, none of these as yet has been linked to the condition in humans. Moreover, the existence of $\gamma-\mathrm{MSH}$ in humans is still a matter of some debate. Nevertheless, the possibility that hormones with both central and peripheral actions like $\gamma$-MSH could cause our salt-sensitivity to fluctuate is an attrac- tive hypothesis to test in a field where salient explanations are scarce.

1. Taubes, G. 1998. The (political) science of salt Science. 281:898-901, 903-907.

2. Ni, X.-P., Pearce, D., Butler, A.A., Cone, R.D., and Humphreys, M.H. 2003. Genetic disruption of $\gamma$-melanocyte-stimulating hormone signaling leads to salt-sensitive hypertension in the mouse. J. Clin. Invest. 111:1251-1258. doi:10.1172/JCI200316993.

3. Lifton, R.P., Gharavi, A.G., and Geller, D.S. 1998 Molecular mechanisms of human hypertension. Cell. 281:545-556.

4. Dahl, L.K., Heine, M., and Tassinari, L. 1962. Role of genetic factors in susceptibility to experimental hypertension due to chronic excess salt ingestion. Nature. 194:480-482.

5. Rapp, J.P. 2000. Genetic analysis of inherited hypertension in the rat. Physiol. Rev. 80:135-172.

6. John, S.W., et al. 1995. Genetic decreases in atrial natriuretic peptide and salt-sensitive hypertension. Science. 267:679-681.

7. Oliver, P.M., et al. 1998. Natriuretic peptide receptor 1 expression influences blood pressures of mice in a dose-dependent manner. Proc. Natl. Acad. Sci. U. S. A. 95:2547-2551.

8. Kennedy, C.R., et al. 1999. Salt-sensitive hypertension and reduced fertility in mice lacking the prostaglandin EP2 receptor. Nat. Med. 5:217-220.

9. Alfie, M.E., Yang, X.P., Hess, F., and Carretero, O.A. 1996. Salt-sensitive hypertension in bradykinin B2 receptor knockout mice. Biochem. Biophys. Res. Commun. 224:625-630.

\title{
Coordinated tumor immunity
}

\section{Glenn Dranoff}

Department of Medical Oncology, Dana-Farber Cancer Institute and Department of Medicine, Brigham and Women's Hospital and Harvard Medical School, Boston, Massachusetts, USA

J. Clin. Invest. 111:1116-1118 (2003). doi:10.1172/JCI200318359.

The most important achievement of cancer immunology thus far may be the development of robust techniques for the identification of tumor antigens $(1,2)$. This work underlies our current understanding that cancer patients frequently generate specific cellular and humoral antitumor responses. Moreover, the expression of

\footnotetext{
Address correspondence to: Glenn Dranoff, Dana-Farber Cancer Institute, Dana 510E, 44 Binney Street, Boston, Massachusetts 02115, USA. Phone: (617) 632-5051; Fax: (617) 632-5167;

E-mail: glenn_dranoff@dfci.harvard.edu. Conflict of interest: The author has declared that no conflict of interest exists.
}

transformation-associated stress genes commonly provokes innate immune reactions (3). Together, these findings unveil a previously unsuspected breadth of immune recognition in tumor bearing hosts.

The characterization of cancer cell antigenicity has fueled efforts to delineate protective immune-effector mechanisms. The task is complicated by the dual role that immunity plays during cancer progression. Recent studies disclosed a marked increase in the incidence of spontaneous and chemicallyinduced tumors in immunodeficient mice compared to littermate controls (4). Since immunocompetent animals efficiently reject tumor transplants from immunodeficient hosts, the experiments support the idea that the immune system functions as an extrinsic tumor suppressor. Consistent with this concept, clinical-pathologic investigations established a strong association between the presence of dense intratumoral $\mathrm{T}$ cell infiltrates and favorable clinical outcomes in patients with malignant melanoma or carcinomas of the colon, kidney, and ovary $(5,6)$.

Other work indicates, though, that tumors may subvert the immune system to facilitate disease progression (7). Unresolved inflammation, whether due to infection, autoimmunity, or environmental agents, markedly increases the risk of cancer. Dysregulated cytokine production promotes cell proliferation and attenuates apoptosis. Phagocyte-derived reactive oxygen species damage DNA. Tumor cell invasion and metastasis exploit the normal cues for leukocyte migration. Collectively, these studies illustrate diverse ways in which the immune system sculpts the hallmarks of cancer (8). 


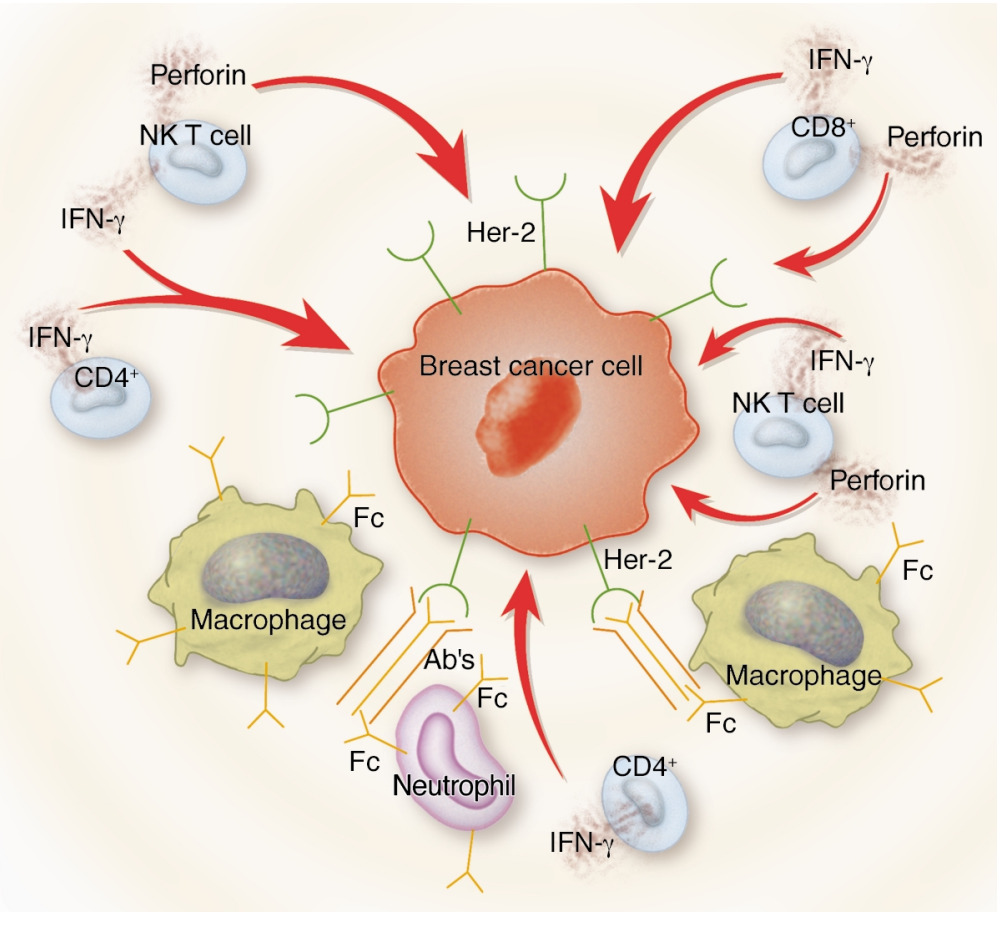

Figure 1

Coordinated tumor immunity. Forni and colleagues (9) show that immune-mediated rejection of established Her- 2 positive breast tumors requires $C D 4^{+}$and $C D 8^{+} T$ cells, CD1d-restricted NKT cells, neutrophils, macrophages, antibodies (Ab's), Fc receptors, IFN- $\gamma$, and perforin.

The dual role of immunity in tumor suppression and progression underscores the possible benefits and risks of cancer immunotherapy. In this issue of the JCI, Forni and colleagues provide important insights into the differing requirements for vaccineinduced elimination of incipient and established tumors (9).

The investigators examined the immune response to Her-2/neu-positive breast carcinomas originating in transgenic mice. Although the tumors express rat Her-2/neu, which differs from the murine homolog by $6 \%$, the model system is intriguing, since Her-2/neu contributes to the pathogenesis of human breast cancer and is a target for clinically efficacious monoclonal antibodies (10). Whereas previous work showed that prophylactic vaccination with tumor cells or DNA encoding Her-2/neu prevented spontaneous tumor formation in transgenic mice $(11,12)$, the current study explores the mechanisms of tumor rejection. Breast cancer cell lines, established from spontaneous tumors, were implanted
Activated neutrophils may lyse cancer cells directly and compromise the tumor vasculature.

Therapeutic vaccination against established tumors proved far more stringent than prevention, and nearly all of the immune components tested contributed to tumor rejection (Figure 1). Successful treatment required a coordinated response involving $\mathrm{CD}^{+}$ and $\mathrm{CD}^{+} \mathrm{T}$ cells, antibodies, Fc receptors, CD1d-restricted NK T cells, macrophages, neutrophils, perforin, and IFN- $\gamma$. Although the basis for this rigorous requirement remains to be clarified, it is tempting to speculate that the rapid growth kinetics of established tumors demand a robust reaction. A large number of tumor cells may also include variants with acquired resistance to some modes of immune attack.

The substantive hurdles to rejecting established tumors suggest that immunotherapies will need to stimulate broad and sustained host responses. Unfortunately, these same requirements also imply that tumors may accomplish immune escape by devising strategies that undermine diverse immune mechanisms. The combination of immunotherapies and other treatments that target resistance pathways may thus be worth exploring.

The study also provides insight into why prophylactic immunization, in which there is considerable redundancy of immune effectors, may be more easily achieved than therapeutic vaccination. Indeed, preventive immunization against pathogen-related tumors already demonstrates great promise for reducing the burden of hepatocellular and cervical carcinoma $(14,15)$. Whereas autoreactivity, as an unintended consequence of immunity to aberrantly expressed self-antigens in tumors, may ultimately limit cancer vaccination, the rules underlying the discrimination of tumor and self are still poorly understood. Carefully conducted vaccine trials in patients at high risk for cancer may shed light on this crucial issue.

\section{Acknowledgments}

This work was supported by NIH grants CA74886, CA39542, and CA66996, the Cancer Research Institute, and the Leukemia and Lymphoma Society. 
1. Boon, T., and van der Bruggen, P. 1996. Human tumor antigens recognized by $\mathrm{T}$ lymphocytes. J. Exp. Med. 183:725-729.

2. Old, L., and Chen, Y.-T. 1998. New paths in human cancer serology. J. Exp. Med. 187:1163-1167.

3. Diefenbach, A., and Raulet, D. 2002. The innate immune response to tumors and its role in the induction of T-cell immunity. Immunol. Rev. 188:9-21.

4. Dunn, G., Bruce, A., Ikeda, H., Old, L., and Schreiber, R. 2002. Cancer immunoediting: from immunosurveillance to tumor escape. Nat. Immunol. 3:991-998.

5. Mihm, M., Clemente, C., and Cascinelli, N. 1996. Tumor infiltrating lymphocytes in lymph node melanoma metastases: a histopathologic prognostic indicator and an expression of local immune response. Lab. Invest. 74:43-47.
6. Zhang, L., et al. 2003. Intratumoral T cells, recur rence, and survival in epithelial ovarian cancer. N. Eng. J. Med. 348:203-213.

7. Coussens, L., and Werb, Z. 2002. Inflammation and cancer. Nature. 420:860-867.

8. Hanahan, D., and Weinberg, R.A. 2000. The hallmarks of cancer. Cell. 100:57-70.

9. Curcio, C., et al. 2003. Nonredundant roles of antibody, cytokines, and perforin in the eradication of established Her-2/neu carcinomas. J. Clin. Invest. 111:1161-1170. doi:10.1172/ JCI200317426.

10. Pegram, M., et al. 1998. Phase II study of receptorenhanced chemosensitivity using recombinant humanized anti-p185HER2/neu monoclonal antibody plus cisplatin in patients with HER2/neu-overexpressing metastatic breast cancer refractory to chemotherapy treatment. J. Clin. Oncol. 16:2659-2671.
11. Rovero, S., et al. 2000. DNA vaccination against rat her-2/Neu p185 more effectively inhibits carcinogenesis than transplantable carcinomas in transgenic BALB/c mice. J. Immunol. 165:5133-5142.

12. Nanni, P., et al. 2001. Combined allogeneic tumor cell vaccination and systemic interleukin 12 prevents mammary carcinogenesis in HER-2/neu transgenic mice. J. Exp. Med. 194:1195-1205

13. Colombo, M.P., et al. 1991. Granulocyte-colony stimulating factor gene suppresses tumorigenicity of a murine adenocarcinoma in vivo. J. Exp. Med. 173:889-897.

14. Chang, M.-H., et al. 1997. Universal hepatitis B vaccination in Taiwan and the incidence of hepatocellular carcinoma in children. N. Eng. J. Med 336:1855-1859.

15. Koutsky, L., et al. 2002. A controlled trial of a human papillomavirus type 16 vaccine. N. Eng. J. Med. 347:1645-1651.

\title{
The fractalkine receptor CX3CR1 is a key mediator of atherogenesis
}

\author{
Myron I. Cybulsky ${ }^{1}$ and Robert A. Hegele ${ }^{2}$ \\ ${ }^{1}$ Department of Laboratory Medicine and Pathobiology, University of Toronto, \\ Toronto General Research Institute, Toronto, Ontario, Canada \\ ${ }^{2}$ Robarts Research Institute, Blackburn Cardiovascular Genetics Laboratory, \\ London, Ontario, Canada \\ J. Clin. Invest. 111:1118-1120 (2003). doi:10.1172/JCI200318237.
}

Recruitment of circulating monocytes to the arterial intima contributes to the formation of atherosclerotic lesions and may participate in their destabilization. Leukocyte emigration from blood into tissues is mediated by multiple adhesion molecules and chemokines, which orchestrate specific steps of emigration and regulate preferential recruitment of different leukocytes depending on their expression patterns of chemokine receptors. Over the last several years, a number of adhesion molecules, including VCAM-1, P-selectin and ICAM-1, the chemokines MCP-1 (also known as CCL2)

\footnotetext{
Address correspondence to: Myron I. Cybulsky, Department of Laboratory Medicine and Pathobiology, University of Toronto, Toronto General Research Institute, UHN, 200 Elizabeth Street, Eaton-4, Toronto, Ontario, Canada M5G 2C4.

Phone: (416) 340-3578; Fax: (416) 340-3578;

E-mail: myron.cybulsky@utoronto.ca.

Conflict of interest: The authors have

declared that no conflict of interest exists.

Nonstandard abbreviations used: single

nucleotide polymorphism (SNP).
}

and IL-8 (also known as CXCL8), and their respective receptors CCR2 and CXCR2, have been functionally implicated in atherosclerosis. Two studies - one recently published in the JCI (1), and the second reported in this issue (2) - expand this list to include the chemokine receptor CX3CR1, the receptor for fractalkine (also known as CX3CL1).

\section{Fractalkine structure and functions}

Among more than 50 known chemokines, fractalkine is the sole member of the CX3C family, and has unique structural and functional attributes (3, $4)$. In contrast to many other chemokines, whose presentation on the cell surface requires interaction with proteoglycans, the $\mathrm{N}$-terminal chemokine domain of fractalkine is anchored to the cell membrane through a contiguous extended mucin-like stalk, transmembrane and cytoplasmic domains (Figure 1). Fractalkine binding to its seven-transmembrane domain $G$ protein-coupled receptor triggers signaling, but it also directly mediates cell adhesion (5). Fractalkine binds CX3CR1 rapidly and firmly, which leads to tethering and arrest of leukocytes under conditions of physiological flow independent of CX3CR1 signaling (5). TNF- $\alpha-$ converting enzyme (also known as ADAM17) can cleave the mucin stalk of fractalkine and release soluble chemokine $(6,7)$. CX3CR1 has two common coding polymorphisms, namely V249I and T280M, that are in strong linkage disequilibrium (almost always occurring on the same allele) and have been associated with interindividual differences in susceptibility to both HIV infection and atherosclerosis (8-10). If replicated, these findings may have clinical relevance.

\section{The role of CX3CR1 in experimental atherogenesis}

The article by Lesnik et al. (1) demonstrated that fractalkine expression was upregulated in atherosclerotic lesions of apolipoprotein $E^{-/-}\left(\right.$apoe $\left.{ }^{-/-}\right)$mice, primarily in intimal smooth muscle cells, which is consistent with the expression pattern observed previously in human atherosclerosis. The function of CX3CR1 in atherosclerosis was assessed by crossing $\mathrm{CX} 3 \mathrm{CR}^{-/-}$mice into the apoe ${ }^{-/-}$ background and feeding these mice a Western-type diet for 5,10 , or 15 weeks. Lesion formation throughout the aorta, including the aortic root, was significantly reduced in the CX3CR $1^{-1}$ groups. These elegant data provide convincing evidence that CX3CR1 plays an important role in experimental atherogenesis, are consistent with a recent 